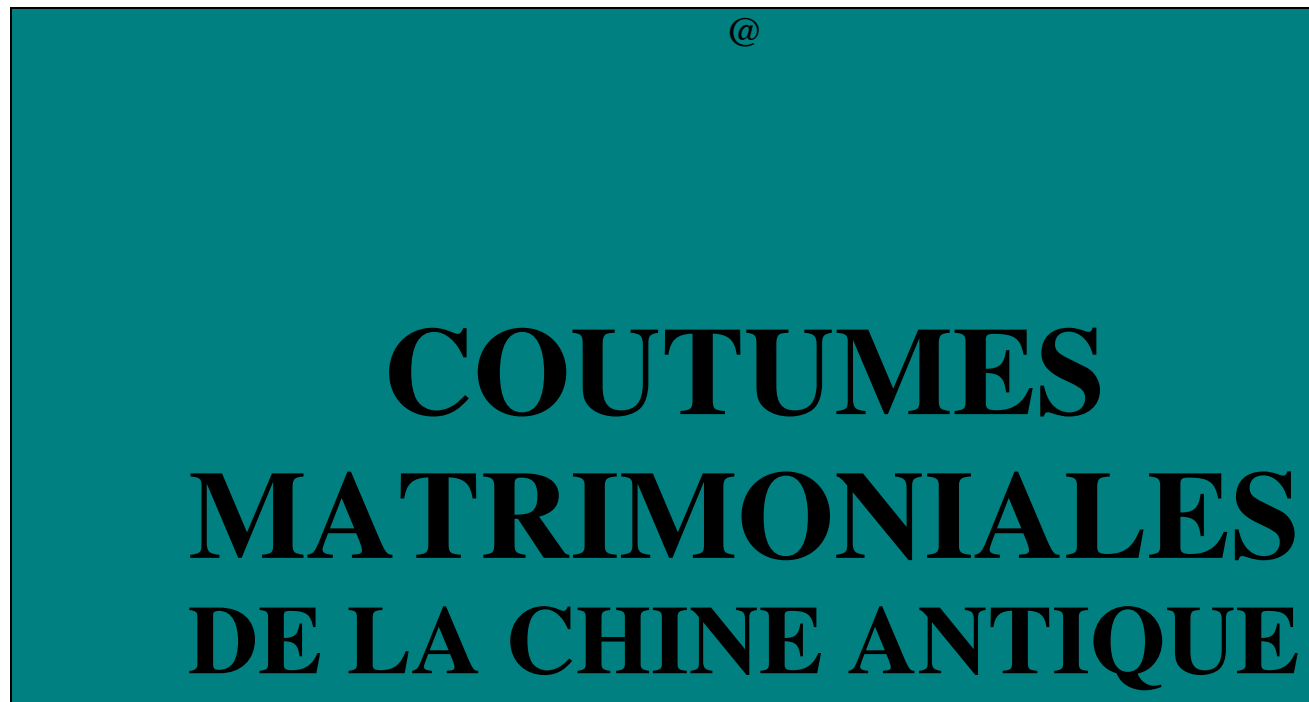

\author{
par \\ Marcel GRANET (1884-1940)
}

1912

Un document produit en version numérique par Pierre Palpant, collaborateur bénévole

Courriel : pierre.palpant@laposte.net

Dans le cadre de la collection : "Les classiques des sciences sociales"

dirigée et fondée par Jean-Marie Tremblay,

professeur de sociologie au Cégep de Chicoutimi

Site web : http ://www.uqac.uquebec.ca/zone30/Classiques_des_sciences_sociales/index.html

Une collection développée en collaboration avec la Bibliothèque

Paul-Émile-Boulet de l'Université du Québec à Chicoutimi

Site web : http ://bibliotheque.uqac.uquebec.ca/index.htm 
Un document produit en version numérique par Pierre Palpant, collaborateur bénévole, Courriel : pierre.palpant@laposte.net

à partir de :

\section{Coutumes matrimoniales de la Chine antique} par Marcel GRANET (1884-1940)

L'article sur les coutumes matrimoniales est paru dans le Toung-pao, vol. XIII, p. 517558, Leyde, 1912.

Textes rassemblés sous le titre «Essais sociologiques sur la Chine», Les Presses universitaires de France, $2^{\mathrm{e}}$ édition, Paris, avril 1990.

Polices de caractères utilisée : Times, 10 et 12 points, et Comic sans MS, 10 points, pour les vers du Cheu king.

Mise en page sur papier format LETTRE ( US letter ), 8.5' $\mathrm{x} 11^{\prime \prime}$

Édition complétée le 30 novembre 2004 à Chicoutimi, Québec.

[ Note css : on circule entre les traductions des chansons du Cheu king de M. Granet (Fêtes et Chansons de la Chine ancienne) et du P. Couvreur (Cheu king) en cliquant sur le signe • placé en titre de la chanson dans chacun des deux ouvrages. De plus un clic sur le nom de la chanson dans Fêtes... envoie sur le site de l' afpc, association française des professeurs de Chinois, contenant la traduction Granet, ainsi que le texte chinois, et si besoin est, la traduction anglaise de J. Legge.] 
Je voudrais grouper ici les renseignements (1) que l'on peut tirer du Che king sur les formes anciennes et populaires du mariage chez les Chinois (2). Avant de reprendre la question pour mon compte, je montrerai comment les auteurs chinois l'ont comprise ; j'indiquerai les raisons du peu de succès de leurs recherches ; je dirai par quelle méthode et avec quels secours on peut essayer de faire mieux. Par ce procédé, on verra de quelle manière il est possible d'utiliser les travaux de la critique chinoise. En outre, et bien que mon intention ne soit pas de faire une étude d'histo ire littéraire, on pourra, chemin faisant, saisir au vif certains procédés de la chanson populaire et l'on aura quelque idée des conditions dans lesquelles elle est née.

C'est surtout pendant les mois d'automne et d'hiver que l'on rencontre dans les villes de la Chine ces cortèges de palanquins, l'un rouge, l'autre vert, de musiciens, d'insignes, de lanternes, qui sont les pompes nuptiales du pays. Naturellement une théorie justifie la pratique : comme le mariage, les deux saisons choisies ressortissent à la catégorie yin; on sait qu'il faut éviter les contaminations et ne pas faire une chose yin à une époque yang.

Or il y a une tradition rituelle d'accord avec la coutume. Le texte principal se trouve dans le Kia yu : "Quand se dépose la gelée blanche, les travaux des femmes sont achevés et les mariages peuvent se faire. Quand les glaces fondent, commencent les travaux des champs et la cueillette des feuilles de

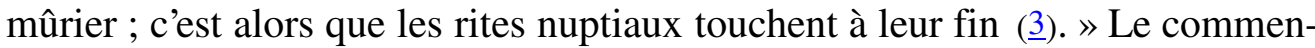
taire précise le sens et donne les garants du Kia yu. Ce sont deux passages du Che king, dont un seul est expressément rappelé dans le texte: "Quand l'homme s'en va chercher femme, c'est avant que les glaces ne soient fondues $(\underline{4}) »$ et « je te prie de ne pas te fâcher, l'automne sera le moment $(\underline{5})$ ». Le commentaire les complète par un texte du Rituel des Tcheou ( $\underline{6}$ ) et il explique que le neuvième mois, où se dépose la gelée blanche, était le début de la saison du mariage, et qu'elle se poursuivait un peu après le commencement des travaux rustiques. "Quand on est arrivé au deuxième mois, les travaux des champs sont déjà commencés ; c'est alors qu'on réunit les garçons et les filles non mariés ; la saison des mariages sans rite (ㄱ) finit ce mois-là. » Ainsi la saison nuptiale déborderait sur le printemps et irait d'un peu après l'équinoxe d'automne à un peu après l'équinoxe de printemps (모.

Or, si l'on examine les passages cités et leurs commentaires, on constate que de nombreux auteurs chinois y ont vu tout autre chose. Notamment le plus illustre des commentateurs, Tcheng K'ang-tch'eng ( $(\underline{)})$, donne le deuxième mois de printemps comme l'époque même des mariages.

Et cette seconde tradition vaut qu'on s'y arrête ; car, si les mariages se font aujourd'hui en hiver ou en automne, ce n'est ni à l'hiver ni à l'automne qu'est 
liée l'idée d'amour, mais au printemps, et presque toujours dans les textes littéraires elle est évoquée par le caractère [].

Quels sont les textes de Tcheng? Le principal est celui auquel faisait allusion le commentaire du Kia yu, un texte du Tcheou li. Une des attributions $\mathrm{du}$ fonctionnaire nommé l'Entremetteur, était, au deuxième mois du printemps, d'ordonner la réunion des filles et des garçons. Pour le commen taire du Kia yи il s'agit là de l'extrême fin de la saison des mariages, et de mariages spéciaux; pour Tcheng, c'est l'ép oque privilégiée des unions matrimoniales. Et il l'explique : «Au milieu du printemps le yin et le yang s'unissent ; pour accomplir les rites du mariage, on obéit aux saisons. » Or l'usage signalé par 1e Tcheou Ii est confirmé par le petit calendrier des Hia : « Au deuxième mois on réjouit en grand nombre les garçons et les filles. » Et c'est encore au deuxième mois, quand reviennent les hiron delles, que se fêtait, dit le Yue ling, le dieu des mariages, le grand Entremetteur.

Entre ces deux théories les écrivains chinois se partagent et leur querelle remplit les commentaires d'un bon nombre de pièces du Che king : il y a peu de chants d'amour ou d'hyménée dans le Kouo fong dont Tcheng et son principal adversaire Wang Sou n’interprètent quelque passage en faveur de leur thèse.

Envisagées du point de vue des commentateurs, ces chansons sont avant tout des compositions morales. Et il ne saurait en être autrement puisque Confucius les admit dans son anthologie.

Mais quelle en est la morale ?

En Chine, la moralité publique est une fonction gouvernementale. - L'ordre parmi les hommes, comme l'ordre dans la nature, sont l'effet du tao tö, de la vertu, du mana, de l'influence efficace de l'empereur. Mais l'empereur délègue de la vertu à l'impératrice et, gardant le tao et lui laissant exercer le tö, il lui confie le département de la moralité sexuelle. La vertu de l'impératrice, c'est de n'être pas jalouse : dès lors, outre que les enfants des autres femmes de l'empereur lui font à elle-même une nombreuse postérité, l'ordre règne dans le gynécée et dans les rel ations des sexes; et les hommes et les femmes de l'Empire s'unissent en temps voulu en de réguliers mariages. Mais si le couple princier est incapable d'exercer sa mission civilisatrice, si le prince est sans vertu, sa femme, qui ne peut avoir qu'une vert u de délégation, ne peut propager les bonnes mœurs : alors hommes et femmes s'unissent sans règles ou laissent passer le temps du mariage.

Tel est le thème que Mao Tch'ang, le plus ancien des commen tateurs du Che king, expose en de nombreuses préfaces. Je cite la plus nette : c'est celle de la sixième pièce du Tcheou nan (10). Cette poésie montre, dit-il, «l'influence de l’impératrice : elle n'était pas jalouse ; aussi les gardons et les filles s'unissaient en temps voulu en de réguliers mariages et il n'y avait pas de célibataires dans l'État ». 
Mais que veut dire se marier en temps voulu ou laisser passer le temps du mariage ? S'agit -il de l'âge des époux ou de la saison des mariages ?

II se trouve dans le $L i k i$ des textes du genre de ceux-ci :

«Un homme à trente ans est dit tchouang (robuste); il est marié » (11) et "Quand une fille a vingt ans elle se marie (12) ». Or ces textes sont interprétés de façons différentes. Pour le Kia yu et Wang Sou, vingt ans et trente ans sont l'âge limite qu'il est interdit de dépasser. Pour Tcheng, c'est à vingt ans précis, à trente ans précis qu'on se mariait (쯔). Quoi qu'il en soit, les hommes à trente ans passés portaient le nom désobligeant de célibataires.

Ceci posé, Wang Sou et les siens raisonnent ainsi : Laisser passer le temps du mariage, c'est ne pas se marier, les filles avant vingt ans, les garçons avant trente ans. Ils insistent sur le fait que Mao Tch'ang, dans la phrase citée plus haut, affirme que, par l’in fluence de limpératrice les mariages se faisant quand il convient, il n'y avait plus de célibataires. Puis ils reviennent au texte du Tcheou li dont Tcheng fait état et en rapprochent un passage immédiatement antérieur : l'Entremetteur des Tcheou « ordonne aux garçons de trente ans de prendre femme, aux filles de vingt ans de prendre mari ». Et ils formulent ainsi leur thèse : Les mariages se contractaient en automne et en hiver; en fin de saison, s'il restait des jeunes gens de vingt et trente ans non mariés, on les unissait d'autorité, in extremis, au deuxième mois de printemps.

Mais la théorie de Tcheng n'est infirmée en rien. Pour lui, laisser passer le temps du mariage, c'est en laisser passer la saison ; mais puisque, d'autre part, on doit se marier à vingt et trente ans précis, c'est aussi, en conséquence, laisser passer l'âge où l'on doit se marier. Et son opinion peut se formuler ainsi : au deuxième mois du printemps, l'Entremetteur officiel, qui possédait l'état civil, dressait la liste des jeunes gens en âge et leur ordonnait de se marier. Il ne reste pour embarrasser Tcheng K'ang -tch'eng que le vers du Che king «l'automne sera le moment » mais, commentant un autre passage (14), il écrit: «au huitième mois le yin et le yang s'unissent, on commence à procéder aux rites du mariage: on annonce le choix, on demande le nom »(15). Ainsi, pour lui, les rites matrimoniaux se divisent en deux groupes; les uns se font à l'équinoxe de printemps, les autres à celui d'automne, aux époques favorables où le yang s'unit au yin.

Et le problème n'avance pas. Aussi les auteurs modernes sont-ils embarrassés, et tranchent-ils la question, brutalement. Ils remarquent dans le Tsouo tchouan de nombreux exemples de mariage en toutes saisons. Ils notent un texte du $L i k i(\underline{16})$ où il est prescrit d'informer le prince du mois où l'on se marie. Ils concluent que l'on se mariait pendant tout le cours de l'année, sauf, peutêtre, parmi le peuple (17). Et ils passent. 
Voilà où nous laissent les commentaires.

Il est difficile d'être juste pour les commentateurs chinois. Ils sont objectifs, clairs, précis, et, sans le moindre développement littéraire, nous font comprendre des textes ardus. Ils lèvent toutes les difficultés de lexique ou de syntaxe et ils font avec une érudition très sûre des rapprochements profitables.

Mais ce sont des philologues bornés. Tout au texte, ils sont incapables d'observation. Ils n'ont point l'idée que la coutume pourrait éclairer l'écrit (18). Ils pensent au contraire que les usages doivent se régler sur leurs textes.

Or, ce sont eux qui interprètent ces textes devenus avec le temps difficiles et obscurs : ils sont des jurisprudents. Et il y a parmi eux des écoles : Tcheng, qui vit à une époque de reconstitution rituelle, interprète strictement. Wang Sou appartient à l'école libérale ; il concilie les textes avec le désir moderne de laisser plus de jeu aux actions humaines (19).

Même ils sont théoriciens et ont leur métaphysique : l'auteur du Kia yu, tout comme Tcheng K'ang-tch'eng, déduit son opinion sur l'époque des mariages de la conception qu'il se fait du yin et du yang.

Voilà bien des raisons de se méfier de leurs interprétations. La plus grave est quils sont historiens et moralistes.

Ils sont fâcheusement historiens. Ils ont la rage de dater et de tout transformer en anecdotes historiques qu’ils rapportent à un personnage bien connu des annales impériales ou seigneuriales. Et, pour montrer que telle chanson blâme ou loue tel prince, ils n'hésitent pas à en expliquer les vers par un symbolisme des plus contournés $(\underline{20})$.

Mais surtout ils sont moralistes. Bien que leurs légendes soient pleines de héros civilisateurs, l’idée confucéenne que tous les péchés contre les rites sont le résultat d'une décadence ne les abandonne jamais (21). Et comme une des prescriptions les plus strictes de leur morale est la séparation des sexes entendue rigoureusement et à la manière des hautes classes de la société de leur temps, ils sont incapables de voir dans les anciens usages de la moralité sexuelle autre chose que des aberrations momentanées, explicables historiquement.

Puisque les commentateurs sont impuissants à nous expliquer les mœurs dont les chansons du Che king sont les témoignages, comment ferons-nous pour les comprendre?

Il nous faut reprendre l'étude du Kouo fong. Nous déterminerons le sens littéral des pièces qui nous intéressent en utilisant les travaux lexicologiques et syntaxiques de la critique chinoise. Mais ce sens littéral une fois établi, nous n'oublierons plus que nous n'avons affaire qu'à de braves chansons populaires. Si le sens aujourd'hui en est obscur, c'est que la langue et les mœurs ont changé ; mais quand on les chantait dans les villages de la vieille 
Chine, elles étaient claires pour tous. Nous laisserons donc de côté tout le symbolisme difficile à quoi oblige l'interprétation officielle et nous nous en tiendrons au sens le plus simple; or, régulièrement, c'est l'interprétation la plus simple qui se trouvera la plus riche et la plus suggestive.

Dans les chansons populaires de tous les pays, certaines associations d'idées reviennent constamment. Si l'on fait bien atten tion au balancement des couplets et aux correspondances des vers, il est facile de dégager ces thèmes ; en confrontant entre elles les chansons, il est possible d'en apercevoir le sens. Nous dégagerons ainsi quelques données positives $(\underline{22})$.

Seulement il est à craindre que ces données soient peu nombreuses. Confucius a singulièrement émondé ce recueil de chansons !

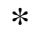

$* *$

Heureusement nous trouverons un secours dans la comparaison.

Le colonel Bonifacy et M. Beauvais ont publié dans le Bulletin de l'École française d'Extrême-Orient d'excellentes notes sur les coutumes des Lolo du Tonkin et des aborigènes de la région de Long-tcheou (23). Or, il est très légitime d'essayer de comprendre les anciennes maurs chinoises à l'aide de celles des populations qui vivent sur le territoire de la Chine ou sur les confins. Ce qu'on nous apprend du mariage chez les t'ou jen de L ong-tcheou, ou sur le culte funéraire des Lolo, nous montre que leurs coutumes sont apparentées de très près aux coutumes chinoises. Il semble même que ces peuples aient dû adopter anciennement la civilisation de la vieille Chine et qu'ils en aient conservé les usages avec plus de fidélité que les Chinois n'ont fait. La comparaison s’impose donc.

Or, ici et là, nos informateurs nous apprennent que des rites sexuels du printemps coexistent normalement avec une réglementation fort stricte du mariage, qui rappelle de très près les rites chinois.

A Long-tcheou, nous retrouvons l'usage de l'entremetteur, des pa -tse, des rites divinatoires, des cadeaux; on prend de multiples précautions pour transporter la fiancée chez le fiancé ; après le mariage, la jeune femme vit recluse pendant trois jours avant d'aller saluer ses beaux -parents. Il semble donc que le mariage soit un événement redoutable et qu'on ne puisse pas sans danger réunir deux sexes qui doivent vivre séparés.

Et cependant M. Beauvais constate que les mours sont fort libres. Et il cite un passage significatif d'un auteur chinois.

«Les jeunes gens et les jeunes filles de cette région aiment à se promener par couples en chantant des chansons. Cette coutume est loin d'être recommandable ; elle n'en est pas moins pratiquée dans toute l'étendue de la province de Yue si (Kouang-si). 
Et ailleurs :

«Chaque année, durant la troisième ou quatrième lune, les jeunes gens et les jeunes filles des différents villages se réunissent pour chanter des chansons à couplets alternés. Les gens des villages voisins viennent, en apportant des provisions, assister à ces sortes de concours. Chaque rassemblement ne comprend pas moins de mille personnes toutes âgées de vingt ans environ. Les indigènes prétendent que, si ces réunions étaient interdites ou empêchées par une cause quelconque, les moissons de l'année ne pourraient arriver à maturité, et de nombreuses maladies épidémiques s'appesantiraient sur ces régions.

Et M. Beauvais ajoute :

«Ces réunions sont souvent prétexte à accordailles. En chantant des chansons à couplets alternés, le jeune homme et la jeune fille qui se font vis-à-vis se déclarent mutuellement leur amour. Il faut avouer que ces réunions donnent lieu à des scènes d'une grande licence. Souvent les couples qui se sont découvert un penchant mutuel s'égarent dans les buissons voisins ou dans les herbes de la jungle pour y prendre les arrhes du futur mariage. Les mandarins chinois n'ont jamais pu interdire pareille coutume. Leur interdiction eût amené à bref délai un soulèvement du pays. »

Ainsi ces rites agraires servent de prélude au mariage et, loin d'être condamnés par la morale publique, ils semblent nécessaires à la prospérité de l’État.

Passons aux Lolo. Eux aussi font usage de l'entremetteur, mais seulem ent après que les jeunes gens se sont accordés. Le mariage apparaît si plein de danger qu'on simule un enlèvement de la fiancée et que les époux commencent par coucher à part. Chez les Man Khoangh, les fiançailles, qui préparent à cet acte si grave, sont fort longues et les fiancés ne doivent pas se parler. Chez les La-qua, en principe, une jeune fille enceinte hors mariage est tuée.

Et cependant les jeunes gens vont chanter sur la montagne. Le premier mois tout entier est consacré aux amours; et c'est une grande fête. Les chansons alors chantées sont dialoguées ; le colonel Bonifacy en donne deux exemples : ce quils ont de plus remarquable, c'est que chaque chanteur considère son partenaire comme un étranger. Et, en effet, au moins chez les La-qua, les jeunes gens d'un même village ne chantent point ensemble. Un ethnographe chinois attribue la même coutume aux Moso (B.E.F.E.-O., VII, 371) : "Quand un couple a chanté avec harmonie, il va s'unir dans les vallées des montagnes ou dans la profondeur des forêts. »

Ici, on ne nous dit pas expressément que ces unions champêtres sont des accordailles. Mais le colonel Bonifacy insiste sur le fait que des couples du 
même village ne chantent pas entre eux ; il y voit une trace d'exogamie entre clans devenus territoriaux (B.E. F. E.-O., VII, 336). Les réunions printanières sont donc bien des rites du mariage.

Ainsi, chez ces populations, les fiancés se choisissent en des joutes de chants d'amour et les accordailles consistent en rites sexuels qui accompagnent une fête agraire du printemps. Et cela n'empêche pas que les mariages ne soient précédés de tout un ensemble de pratiques destinées à atténuer les effets redoutables du rapprochement des sexes.

$$
\begin{gathered}
* \\
* *
\end{gathered}
$$

Nous voici armés pour lire les chants amoureux du Che king.

Commençons par la dernière des chansons (24) du Tcheng fong (Couvreur, p. ${ }_{101}$ ) [Cf. Fêtes et Chansons..., LII] et ne tenons pas compte de la préface qui y voit une satire et qui explique la débauche des jeunes gens et des chansons par dincessants troubles militaires (25).

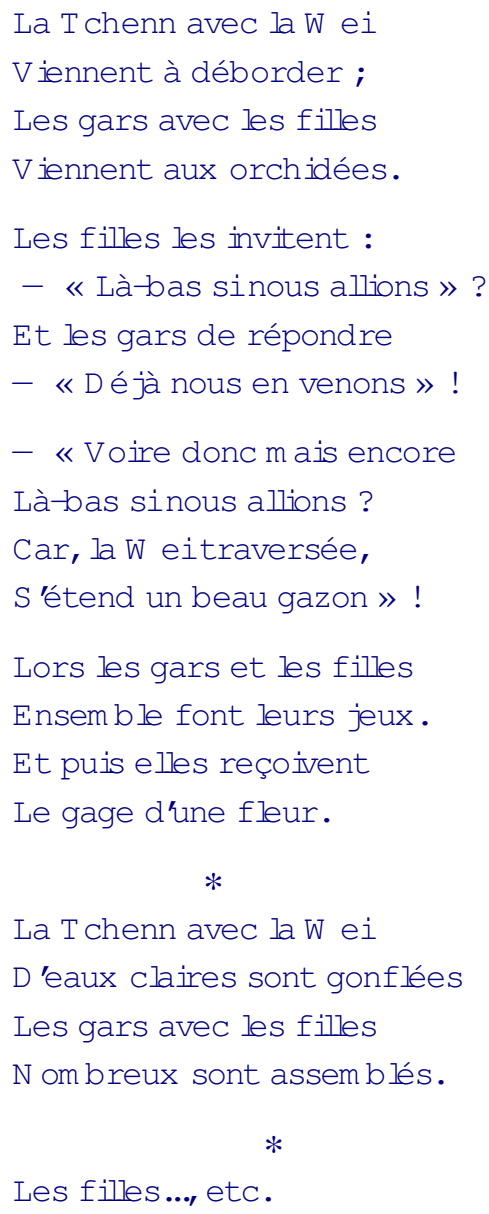


Le parallélisme, deux à deux, des vers des couplets nous indique que la cueillette en commun des orchidées est liée à la crue des rivières. Elle a lieu, dit le commentaire, au milieu du printemps à la fonte des glaces. Alors les jeunes gens non mariés, émus par le printemps, vont cueillir une fleur odorante et se livrer à la débauche.

Mais que signifie cette fleur odorante ? Un texte cité dans le dictionnaire de K'ang -hi, à l'occasion de cette chanson, nous dit qu'elle avait le pouvoir de détruire les pestilences et d'écarter les mauvaises influences (26). Quels dangers veut-on donc écarter?

Les commentaires identifient la plante [] et la plante []. Or le petit calendrier des Hia nous apprend qu'on recueillait la dernière, au cinquième mois, et les Commentaires font voir qu'on la portait à la ceinture. En effet, au chapitre «Nei tsö » du Li ki nous voyons que les femmes mariées et les jeunes filles, même les garçons qui ne sont pas hommes faits, portaient suspendus à la ceinture des sachets de parfums (27). Or au même chapitre il est dit, parmi les précautions rituelles que prend une concubine avant de coucher avec son maître, qu'elle doit attacher à sa cein ture un sachet de parfums. Il n'y a pas de doute sur la signification de la cueillette; garçons et filles se munissent de la plante odorante afin d'écarter les dangers qu'ils s'en vont courir.

Ainsi gardés, ils se provoquent en vers alternés. Les commentateurs chinois remarquent que les filles ont l'initiative et qu'elles insistent. Les garçons se décident. Les couples vont s’isoler par delà la rivière, et font acte de mari et de femme. Puis on se sépare et les garçons font cadeau aux filles de la fleur [], ce qui sert à lier amitié.

Il est difficile didentifier la fleur, mais le rôle qu'elle joue est clair, surtout, si l'on rapproche (28), comme le font les auteurs chinois, le caractère [] du caractère [] qui n'en diffère que par la clef de la soie : elle donne liidée de lier et [] signifie convention. Notre fleur est donc celle qui sert de gage (29). Si les garçons la donnent aux filles, c'est en vertu de la conven tion qu'ils viennent de conclure; elle est le signe des accordailles ; les jeunes gens ont lié amitié, ou encore, selon d'autres expressions parallèles, lié parenté, lié parole, et toutes ces expressions signifient se fiancer. Mais il faut noter que, cette fleur donnée, les jeunes gens se séparent et qu'on l'appelle la plante des séparations.

Ainsi les jeunes gens du pays de Tcheng, comme les t'ou jen et les Lolo se réunissent en grand nombre et, après avoir chanté ensemble, s'en vont, en guise d'accordailles, s'unir au milieu des champs. Ils n e vont point «dans la profondeur des forêts », mais sur les beaux gazons « d'au delà la Wei », et si j'ai traduit, en insistant, «la Wei traversée », c'est que le passage de la rivière est un des thèmes des chansons amoureuses du Che king, le plus important et le plus significatif. 
La chanson (으) «jupe troussée », de la même section Tcheng, rappelle aux commentateurs je ne sais quel incident de politique seigneuriale. Lisons-la simplement :

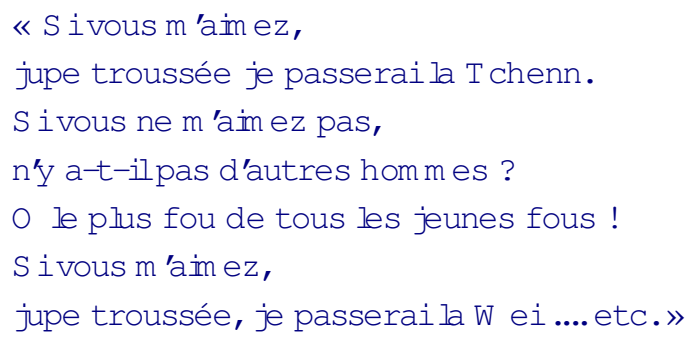

Je demande comment l'on peut trouver dans ces vers autre chose que la chanson provocante d'une jeune fille. Surtout, si l'on a lu les deux pièces que je traduirai maintenant. $\bullet$ L'une est $1 \mathrm{la} 9^{\mathrm{e}}$ du Pei fong (Couvreur, p. 38) [Cf. Fêtes et Chansons..., L ] et les commentateurs affirment quil y est question d'un adultère seigneurial.

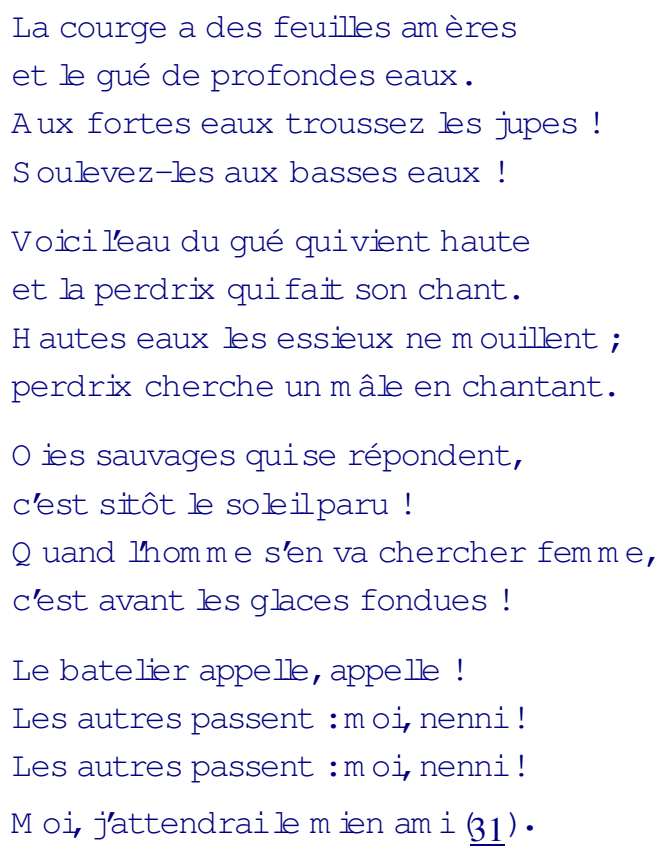

Pour Tcheng K'ang -tch'eng la scène se passe à l'automne parce que la courge a des feuilles amères. Cette opinion est inadmissible; ce n'est pas en automne que la perdrix cherche à s'accoupler ( $\underline{32})$; c'est au printemps, à la saison même où l'eau des rivières vient haute et où les jeunes filles la passent avec leurs amis.

Pourquoi donc parle-t-on de la courge ? C'est certainement une allus ion à la calebasse nuptiale qui, séparée en deux moitiés, sert de coupe aux époux (3ㅡ). Et ce rappel d'un détail du rituel des noces n'est pas le seul de la chanson. L'oie sauvage est offerte, en effet, le Yi li confirme le fait, à diverses 
cérémonies des fiançailles, sitôt après le lever du soleil. C'est seulement le jour des noces que le fiancé offre l'oie rituelle au crépuscule du soir (느). Pour Tcheng, «quand l'homme s'en va chercher femme »doit aussi s'entendre d'une cérémonie des fiançailles où l'on prie de fixer la date du mariage. Le vers est difficile. Le caractère [], qu'on identifie à [], désigne, normalement, la pompe nuptiale, et a, normalement, la fiancée comme sujet. Par exemple, on dit fréquemment (프) dans les chansons d'hyménée : cette fille va à la maison du fiancé, cette fille se marie. Pour expliquer le mot, il faut lui donner ici une valeur causative : et comprendre, dit Tcheng K'ang -tch'eng, faire en sorte que la jeune fille vienne chez soi. Mais alors le mot a l'air de perdre son sens tech nique et sa précision. Aussi Tcheng voit-il dans le vers une allusion non à la pompe nuptiale mais à une des cérémonies des fiançailles. Il semble donc que le vers parle simplement des démarches par lesquelles on prépare un mariage : aussi l'ai -je traduit: quand l'homme s'en va chercher femme ( $\underline{36})$.

Un autre vers fait difficulté : «la perdrix cherche un mâle en chantant ». Le caractère [] désigne normalement le mâle d'un quadrupède (37). Les commentateurs se sont jetés sur ce sens; et le P. Couvreur traduit d'après eux « la faisane crie et pour compagnon on lui cherche un quadrupède ». De ce sens, évidemment, on peut tirer de belles réflexions morales. Mais c'est ne pas apercevoir que ce $8^{\mathrm{e}}$ vers correspond au $16^{\mathrm{e}}$. La jeune fille comme la perdrix cherchent à s'accoupler; de même que la jeune fille qui chante la chanson

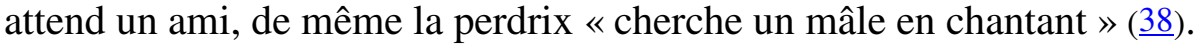

En fait, nous nous trouvons ici en présence d'un nouveau thème des chansons d'amour : celui du chant des oiseaux au printemps. Le chant du loriot, particulièrement, est lié aux rites nuptiaux. «Le loriot vole, — ses ailes sont brillantes » (39). Et ailleurs : «Les journées de printemps s'attiédissent ; - voici que chante le loriot (무). » Le chant du loriot signale le printemps dans les vieux calendriers. Au deuxième mois de printemps, dit celui des Hia, «voici que chante le loriot». Et le Yue ling : «La pluie commence à tomber, le pêcher commence à fleurir, le loriot chante (1ㅡ). »

Ainsi, avant de s'aller débaucher dans les champs, comme disent les auteurs chinois, les jeunes filles rappelaient dans les vers de leurs chansons les rites nuptiaux, et, vraiment, cela ne s'expliquerait guère si les réunions printanières ne leur avaient point paru des rites, tout aussi obligatoires que les autres.

La chanson $\mathrm{n}^{\circ} 9 \mathrm{du}$ Tcheou nan (느) est plus difficile à comprendre avec précision. Elle a pour titre «l'étendue de la rivière Han » et la préface nous dit que la vertu aussi s'étend loin ; grâce à celle du roi Wen qui a transformé les principautés du midi, au confluent de la rivière Han et du Yang tseu, les jeunes gens ne violent plus les rites. Si les commentateurs étaient moins moralistes et plus observateurs, ils auraient pu savoir ce qu'un ethnographe chinois nous apprend, à propos des Fo yi qui n'aiment point les filles vierges $(\underline{43})$ : 
«De même que dans le pays du confluent du (Yang-tseu) kiang et de la rivière Han, ils leur laissent toute liberté de se promener (cf. le troisième vers de la chanson) et ne leur défendent de sortir qu'à l'âge de la puberté.

Voici donc la chanson :

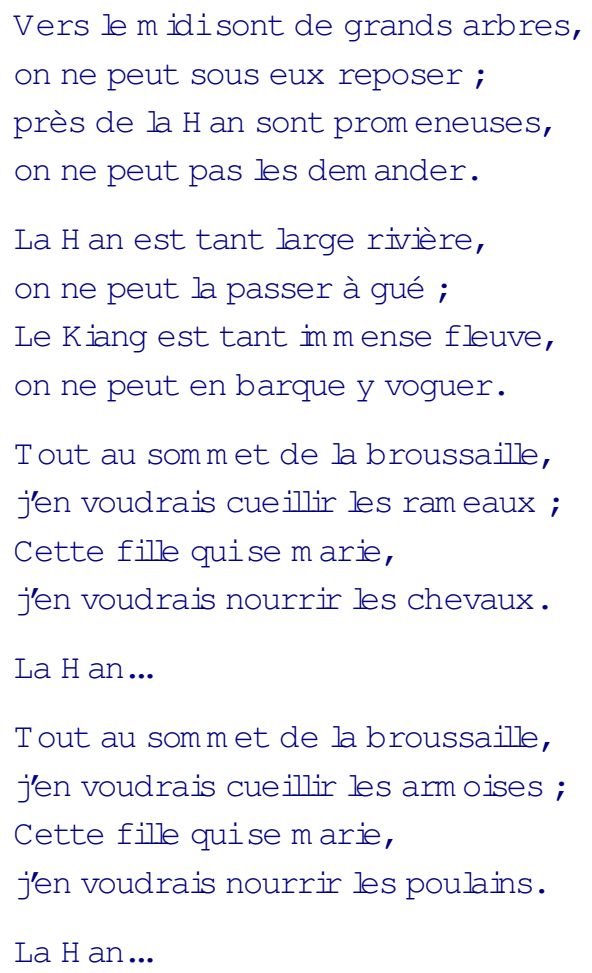

Ici, encore, comme dans la chanson de la Courge, le thème printanier du passage de la rivière est intimement uni à des rappels d'usages nuptiaux, tels que celui des chevaux du char de la mariée. J'ai cependant l'impression que cette chanson est plutôt un chant d'hyménée qu'un chant printanier ; il me semble que, comme dans la première chanson du Chao nan (Couv., p. $\bullet{ }_{166}$ ) [Cf. Fêtes et Chansons..., IX] où revient aussi le vers «cette fille se marie », le thème principal est le cortège de la mariée. Si j'ai raison, nous avons ici la contre-partie de la chanson précédente : un chant nuptial où sont rappelés, sans la moindre gêne, les rites du printemps.

Quoi qu'il en soit, nous trouvons dans cette pièce deux nou veaux thèmes : celui des bosquets au sud des villes et celui des fagots dherbes ou de branchages.

Il semble que c'était de préférence à l'est ou au sud des murailles, sur des tertres ombragés que les jeunes gens se donnaient rendez-vous. Cela apparaît dans la $1^{\mathrm{e}}$ et la $2^{\mathrm{e}}$ chanson du Tch'en fong (Couv., p. ${ }^{145}$ ) [Cf. Fêtes et 


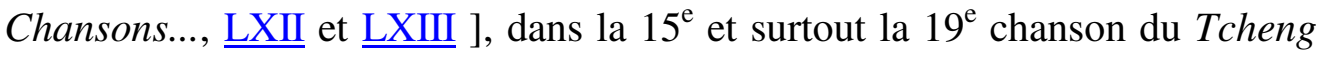
fong (Couv., p.• ${ }_{97}$ et ${ }_{100}$ ) [Cf. Fêtes et Chansons..., XXIII ]. On dansait à ces réunions, qui avaient lieu par une belle matinée, on allait en bandes se promener, on chantait et l'on finis sait par s'offrir des fleurs, et nous savons ce que cela veut dire.

Le thème des fagots apparaît plutôt dans les chants d'hyménée: il est particulièrement net dans la $5^{\mathrm{e}}$ chanson du T'ang fong (4ㅗ) qui s'appelle « le fagot lié ». Il n'est pas facile de voir à quoi ce thème correspond $(\underline{45})$ : allusion à la provende des chevaux ? aux flambeaux nuptiaux ? Ces explications ne satisfont guère.

Faudrait-il voir dans le fagot lié un symbole du contrat matrimonial ? (On se rappelle l'emploi du mot lier dans les expressions qui signifient se fiancer.) Cela serait possible, puisque les fagots et la hache qui sert à les couper sont associés fréquemment à l'idée d'entremetteur (46).

Mais je croirais volontiers que ce symbolisme est récent et j'ai plus de penchant pour une autre hypothèse. On a vu dans la chanson traduite que de l'armoise était cueillie. Or l'armoise n'est pas une plante vulgaire ; on l'emploie dans le culte des ancêtres (그). D'autre part, c'est un usage chinois de suspendre aux maisons que menace quelque influence maligne un bouquet de plantes efficaces. Enfin c'est une coutume, bien connue des ethnographes, que les habitations où se passe un événement redoutable, un accouchement par exemple, sont mises en interdit et signalées par quelque objet convenu. Ainsi les anciens Chinois suspendaient-ils à la porte de l'accouchée des flèches ou une serviette ( $\underline{48})$. Les fagots protégeraient-ils et signaleraient-ils la maison nuptiale $(\underline{49})$ ?

Venons-en à la chanson 12 du Chao nan (Couvreur, p.• ${ }_{26}$ ) [Cf. Fêtes et Chansons..., LXIV].

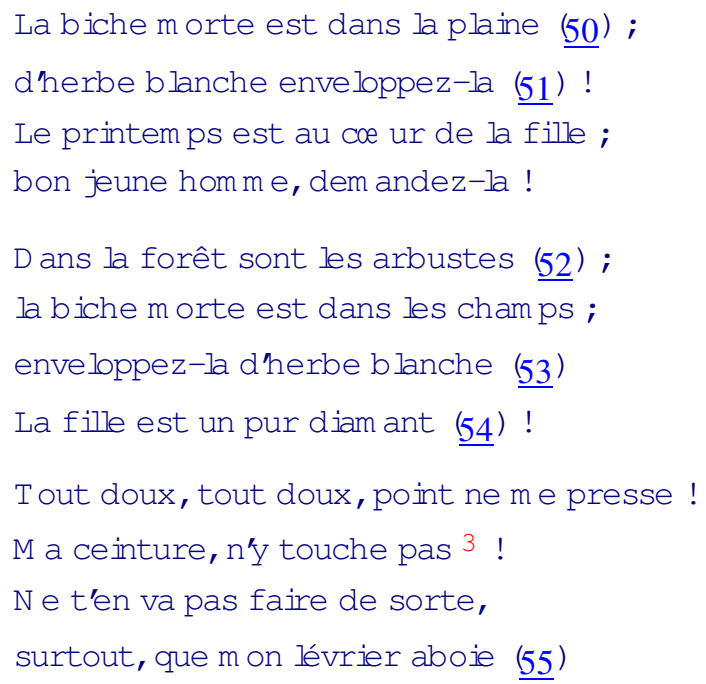


- Remarquons tout de suite l'emploi du mot printemps ; il est très voisin de celui qu'en font les auteurs modernes. Mais le commentaire précise : avoir le printemps dans son coar, c'est rêver au second mois de printemps, saison des amours.

Dès lors, le sens de la chanson apparaît bien. Le printemps et ses fêtes sont passés ; la jeune fille invite son ami à la demander à ses parents, à leur faire la présentation rituelle de la double peau de cerf, et avant que ces rites ne soient accomplis, elle se refuse au jeune homme trop pressé.

- J'ai rendu par ceinture un mot qui exactement signifie serviette. C'est une pièce tellement importante du costume féminin qu'on la suspend à la porte pour signifier qu'une fille est née $(\underline{56})$. - Au jour du mariage, la mère de la fiancée la lui attachait à la ceinture (57). Le Yi li nous apprend que c'était au moment de lui donner les suprêmes instructions (마). Et la gouvernante de la jeune femme, quand les deux époux s'étaient dévêtus, la lui rendait (59), car, dit le commentaire, elle s'en servait « pour se purifier ». «Ne touche pas à ma

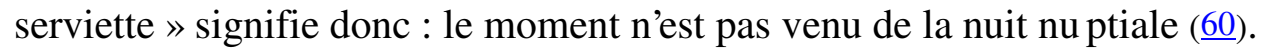

Nous retrouvons une situation analogue, plus développée, dans une jolie romance, mais impossible à rendre en français, qui est une complainte de la mal mariée, et qui s'intitule : le Rustre ; c'est la $4^{\mathrm{e}}$ du Wei fong (Couvreur, p.• ${ }_{67}$ ) [Cf. Fêtes et Chansons..., LXVI ].. Elle se compose de 6 laisses de 10 vers, dont je traduis les deux premières, qui nous racontent un mariage paysan.

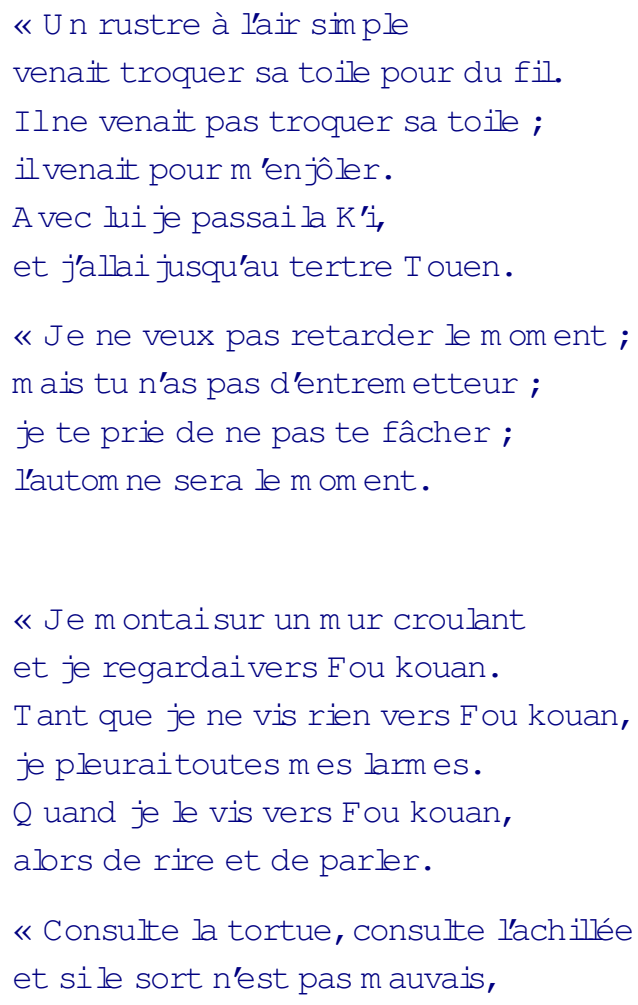


viens t'en avec ta voiture,

nous y porterons $m$ on trousseau.

Les faits contés dans la deuxième laisse se passent à l'automne, mais à quelle époque ceux de la première ?

- Pour Tcheng K'ang-tch'eng, c'est au quatrième mois de l'année que l'on commençait à vendre les fils de soie. Tcheng s'appuie sur le Yue ling : or, il y est dit que c'est seulement au quatrième mois que l'éducation des cocons est terminée. Lỉmpôt qu'on lève alors est un tribut en cocons. De plus, il est ici question de pièces tissées : et le Yue ling nous dit qu'elles n'étaient prêtes pour la teinture qu'au troisième mois de l'été. Il faut cependant que la scène de cette première strophe se passe assez longtemps avant l'automne puisque la deuxième nous décrit une longue attente.

En fait, un texte cité dans le Dictionnaire du P. Couvreur, nous apprend que l'on vendait du fil de soie nouveau dès le deuxième mois. De plus, la deuxième chanson du Tch'en fong (Couv., p. • ${ }_{145}$ ) [Cf. Fêtes et Chansons..., LXIII ], nous dit que les filles cessaient de filer pour aller aux réunions printanières. Mais ce qui, pour moi, donne la date avec certitude, ce sont les $5^{\mathrm{e}}$ et $6^{\mathrm{e}}$ vers : «avec lui je passai la $\mathrm{K}$ ’ — et j'allai jusqu'au tertre Touen ». Si l'on se rappelle ce que signifient le thème du passage de la rivière - qui revient encore à la $4^{\mathrm{e}}$ laisse $(\underline{61})$ - et celui des réunions sur les tertres ombragés, l'hésitation n'est pas possible.

- C'est au printemps, quand cessent les travaux dintérieur, quand le peuple sort des maisons et se disperse, que doit se faire le colportage. Les jeunes gens des villages voisins (62) se rencontrent alors. Notre jeune fille a écouté les propositions d'un jeune étranger et l'a suivi à une réunion printanière ; puis il leur faut se séparer ; il leur faut laisser passer l'été ; alors, le jeune homme pourra demander la fille à sa famille en lui envoyant un entremetteur (63). L'automne est attendu avec impatience, mais l'ami est fidèle à ses promesses, les sorts sont favorables, et la jeune fille monte dans la voiture nuptiale.

Outre que l'on voit bien dans cette chanson que les libres accordailles printanières sont suivies, à l'automne venu, des rites classiques du mariage, elle a le grand mérite de rappeler notre attention sur le rythme de la vie agricole.

On sait que le Kia yu y insistait: "quand se dépose la gelée blanche, les travaux des femmes sont terminés et les mariages peuvent se faire ; quand les glaces fondent, commencent les travaux des champs et la cueillette des feuilles de mûriers ; c'est alors que les rites nuptiaux touchent à leur fin ». Il suffit, en effet, de lire le Yue ling pour apercevoir dans la vie sociale des Chinois des variations saisonnières très marquées. Les deux dates critiques sont les périodes qui suivent le milieu de l'automne et le milieu du printemps. Elles sont indiquées dans le calendrier agricole par le dépôt de la rosée et celui 
du givre. Et l'une de ces dates est aujourd'hui encore l'un des 24 termes de l'année chinoise. Or, c'est un thème des chansons d'amour et d'hyménée que le dépôt de la rosée. C'est au moment où la rosée commence à se déposer sur les plantes des champs, que les jeunes filles font «la rencontre» de leurs amants (64); mais quand la rosée est trop abondante sur les chemins, il y a un empêchement tel aux rites nuptiaux qu'il peut sinvoquer en justice (65).

- Donc le cycle agricole était inauguré par une grande fête champêtre où, comme chez les Lolo et les t'ou jen, se contrac taient les fiançailles. Puis, les champs une fois fécondés ( $\underline{66}$ ), les travaux rustiques commençaient et, les hommes aux champs, les femmes aux vers à soie, c'était la séparation des sexes, à la paysanne. Mais quand, vers le neuvième mois, la gelée blanche se dépose, les travaux cessent (67), tout le monde rentre dans les maisons, le grillon se cache sous le lit (68); les sexes se trouvent réunis, et les mariages sont consommés.

Ainsi la longue saison des travaux champêtres s'étend entre les accordailles printanières et la réunion définitive des époux. Pourquoi donc ces longues fiançailles, qui séparent les fiancés?

D'abord, elles sont une préparation qui diminue les dangers si redoutables de l'union sexuelle. Les jeunes gens se sont unis une première fois, dans une fête sacrée, sous les meilleurs auspices ; mais toutes les craintes ne sont pas dissipées. Si l'on veut sentir combien elles sont vives, qu'on se rappelle les précautions accumulées aux jours d'hyménée, qu'on songe aux lamentations des jeunes filles t'ou jen qui se considèrent comme mortes (69), et qu'on prenne le $L i k i$ : on y verra qu'aux mariages « une pensée sombre et affligeante occupait les esprits » (무) et $\bullet$ qu'on laissait dans la maison de la fiancée tes flambeaux allumés durant trois nuits (1ㅡ) comme s’il y avait eu un mort. On comprendra alors qu'on se soit préparé au mariage par des purifications et des abstinences (2) et que la jeune fille en y songeant fût triste (73). Aussi fallait-il peu à peu préparer les esprits à cet acte si grave. Pour qu'un homme s'approprie définitivement une femme, le rite, si sacré soit-il, des accordailles est insuffisant; il faut encore que les familles y consentent et que des rites publics habituent la société et les fiancés eux-mêmes à un tel changement d'état civil.

Mais aussi, l'union sexuelle en dehors des fêtes qui la consacrent, apparaît comme tellement impure qu'il n'en faut point contamin er les choses fastes comme le travail (4). On ne se marie point pendant la saison des travaux et, réciproquement, après le mariage on ne saurait travailler. Un fonctionnaire ne peut exercer sa charge pendant un an sil se marie, tout comme s'il perd son père ou sa mère $(\underline{75})$. La nouvelle épouse ne doit rien faire de trois mois $(\underline{76})$ : ce n'est qu'au bout de ce temps d'interdit, une saison, qu'elle est enfin assez pure pour travailler, comme pour se présenter devant les ancêtres (77).

Voilà pourquoi l'on se fiance au printemps et l'on se marie à l'automne. 
Ainsi, par l'analyse et le rapprochement des textes du Che king, et par l'usage de la comparaison, nous arrivons à une opinion qui diffère de celles des auteurs chinois. Nous ne nous en étonnerons pas puisque nous avons vu qu'il y a des raisons théo riques de se méfier d'eux. Mais notre interprétation recevrait une nouvelle force si nous pouvions faire sentir pourquoi, dans l'espèce, les recherches de la critique chinoise n'ont pas abouti. Comme la raison essentielle s'en trouve dans la diffusion de la morale confucéenne - ou plus exactement de la morale des hautes classes féodales — nous montrerons du même coup comment se sont modifiées les mours sexuelles (마).

En premier lieu, les rites agraires populaires ont dû très rapidement passer pour des superstitions : les rituels nous parlent un peu longuement d'une seule de ces fêtes ; encore en parlent-ils en termes fort obscurs. La centralisation féodale, puis impériale, a fait du prince le chef religieux suprême ; lui seul était chargé des rites qui rendaient les années prospères. On conçut bientôt que l'ordre de la nature ne dépen dait que de sa vertu. Et les commentateurs, imbus de cette idée, ne pouvaient comprendre le sens des fêtes printanières, d'autant qu'elles se mêlaient de rites sexuels que leur morale tenait pour abominables !

En second lieu, leur idéal moral est, naturellement, celui des classes nobles. Or, on est noble quand on a des ancêtres qu'on peut célébrer par un culte, et le fond de la morale, ce sont les règles rituelles qui dérivent du culte des ancêtres. Aussi le mariage noble diffère-t-il du populaire. Il a pour fin de donner à un homme le moyen de rendre à ses pères les honneurs cultuels, en lui adjoignant la collaboratrice, qui est nécessaire. Les fiançailles, alors, sont le temps pendant lequel la jeune fille fait l'apprentissage des rites dans le temple familial. Pendant cette période elle était sévèrement recluse (9ㅜ); à moins de raison très grave nul n'entrait chez elle. Aussi, peu à peu, un idéal de chasteté s'élabora. Dans les classes riches rien n'obligeait les femmes à se mêler à la vie commune; le bon ton, la règle furent que leur vie se passait dans la partie la plus reculée de la maison; on les y gardait soigneusement (으). Alors la formule de la séparation des sexes fut observée dans le sens le plus strict. La $9^{\mathrm{e}}$ chanson du Wang fong (1ㅡ) en donne magnifiquement la formule : "Vivants, nous n'aurons pas la même chambre ; et morts nous aurons un même tombeau. » L'opposition des sexes, dès l'origine, rendait redoutable l'uni on sexuelle; on en vint à interdire tout commerce des hommes et des femmes : - l'entremetteur, d'ambassadeur qu'il était, devint un apparieur ; sans son intervention un homme ne pouvait connaître d'une femme même son nom (2). Et la vie chaste et retirée du gynécée sembla la préparation convenable et nécessaire au mariage, comme autrefois les licences sexuelles du printemps paraissaient atténuer les dangers de la réunion définitive.

Comment les commentateurs, accoutumés aux règles nouvelles, pouvaient-ils comprendre les libres fiançailles d'autre fois? 
Seulement les rites par lesquels s'unissaient les nobles, devenus plus imposants parce qu'ils étaient accomplis par des personn ages considérables, se retrouvaient dans le Che king; il était donc naturel de penser que le mariage antique ne différait point du moderne $(\underline{83})$ et les libertés printanières devaient apparaître comme des manquements aux rites.

Enfin les commentateurs étaient profondément pénétrés des principes de la centralisation administrative. Cela explique leur confiance dans le Tcheou $l i$ et qu'ils s'y soient trompés. Le carac tère administratif de ce texte révèle assez la basse époque de sa rédaction ; tout y est rapporté à un département spécial de l'administration impériale, à un fonctionnaire déterminé. Quand il est dit que l'entremetteur « ordonne de réunir les garçons et les filles », il est difficile de comprendre qu'il s'agit là d'une cou tume $(\underline{84})$ régularisée et non d'une mesure administrative (5ㅡ). Aussi Tcheng croit-il que ce fonctionnaire de l'État-civil a pour fonction d'unir les jeunes gens en âge à (époque favorable, et Wang Sou qu'il est chargé de veiller à ce que les célibataires récalcitrants ne se soustraient pas aux obligations matrimoniales.

Un seul problème pouvait se poser aux auteurs chinois, celui de la saison du mariage, car ils sentaient profondément qu'un calendrier est une loi qui importe à l'État $(\underline{86})$.

Mais, ici encore, une cause d'erreur viciait leurs recherches. Lettrés de l'époque impériale, ils ne connaissent que le calendrier officiel, calendrier savant, à base astronomique ; philosophes, ils ont raisonné sur le yin et le yang jusqu’à faire sortir toute une métaphysique de ces classifications primitives. Or le calendrier du Che king est un calendrier agricole (7). Ce sont des observations paysannes qui y marquent les dates; et les saisons ne s'y distinguent pas avec une précision absolue. Le temps des travaux peut se prolonger plus loin que le neuvième mois ( $\underline{88})$ et les fêtes des accordailles se poursuivre un peu tard dans le printemps $(\underline{89})$.

En fait, c'étaient les fêtes qui marquaient les dates critiques, la fête du printemps, que nous connaissons, et celle du repos des laboureurs que le Yue ling fixe au dixième mois $(\underline{90})$. La saison de la dissémination et des travaux, celle du repos et de la vie urbaine ou villageoise, s'opposaient de la façon dont les commentateurs opposent le yin et le yang, et les époques où, pour eux, le yin et le yang s'unissent, c'étaient les points critiques, les période s de fêtes. Mais lorsqu'on ne sentit plus le lien de ces notions primitives et du rythme social qu'elles exprimaient, quand le yin et le yang furent devenus des principes cosmogoniques, chaque école philosophique put déduire de l’idée particulière qu'elle s'en faisait une théorie pour fixer la saison du mariage, puisqu'on sentait encore qu'il dépendait du calendrier. Niais ces théories ne pouvaient rendre compte des faits dans la mesure même où les deux grands principes, vidés de leur contenu de représentations anciennes, n'étaient plus que les entités dont usait une astronomie déjà savante (1ㅡ). 
En déterminant comment s'expliquent les théories des écri vains chinois, nous avons appris l'histoire des variations des coutumes matrimoniales dans l'ancienne Chine. Elle peut se résumer dans celle des sens du mot [].

Il apparaît une fois dans le Che king, dans une pièce du Wang fong (92). Il y est question d'un fonctionnaire en tournée qui vient au nom de l'emper eur exercer l'influence civilisatrice et réformer les mours ; quand il arrive, les jeunes filles se lamentent de ne plus pouvoir rejoindre les garçons. Il s'agit bien ici des réunions printanières, et le mot [] signifie s'unir dans les champs. D'ailleurs, si l'on peut avoir confiance dans l'étymologie, celle de [], un homme dans les herbes, serait significative.

Dans le Tcheou li, à une nuance près, le sens est le même ; après avoir indiqué la mesure que l'entremetteur prend, au deuxième mois, d'unir les garçons et les filles, le texte ajoute : «(alors) il n'est pas défendu de s 'unir dans les champs » (93). Mais on sent la défaveur.

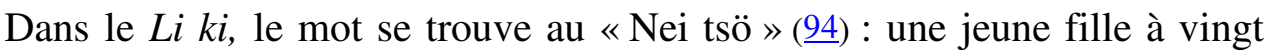
ans est mariée... «si elle a été demandée en mariage, elle est épouse ; si elle s'est mariée sans rites, elle est concubine ». Le sens est ici d'une union inférieure conclue sans rite, sans entremetteur surtout, et qui ne produit pas les effets du véritable mariage.

Enfin, dans les préfaces de Mao Tch'ang et pour les commen tateurs, le mot désigne les rapports débauchés des jeunes gens.

Il ne me reste, pour conclure après ces analyses, qu'à pré senter les faits d'ensemble.

Au deuxième mois de printemps, aussitôt que le yang de retour ramène les hirondelles et la chaleur, dès que, sur les plantes des champs, la rosée remplace la gelée blanche, lorsque la glace se met à fondre et que vient la première crue des rivières, quand les fleurs précoces poussent dans les coins humides et que les oiseaux chantent et cherchent à s'accoupler, — alors, les hommes sortent de leurs maisons, les filles cessent de filer, et les garçons, de village en village, colportent la toile tissée. Émus par le printemps, garçons et filles s'en vont en bande, à l'est ou au sud des murailles, danser sur quelque tertre bien exposé, à l'ombre des arbres. Puis, le long des rivières gonflées, dans les prairies basses, ils s'en viennent cueillir la fleur odorante qui est un encens capable d'écarter les puissances malignes et que l'on porte dans un sachet pendu. à la ceinture. Filles et garons chantent et se provoquent et se choisissent. Jupes troussées, on passe la rivière ; les couples sisolent et, les commentaires parlent ainsi, font acte de mari et de femme. Alors, les filles reçoivent des garçons la fleur que l'on donne quand on se sépare, comme gage d'une réunion prochaine. Et les accordailles sont faites. 
Mais je crois bien qu'il leur fallait passer l'été séparés, chacun dans son village. A l'automne, les parents avertis envoient un entremetteur ; au soleil levant, l'oie sauvage est offerte, puis la double peau de cerf ; et, au jour favorable fixé par la tortue ou, les bâtonnets d'achillée, lorsque la mère a noué la ceinture de la, fiancée et y a attaché la serviette rituelle, la jeune fille monte, à la nuit tombante, dans la voiture nuptiale, et va boire avec son époux aux deux moitiés de calebasse.

Ainsi les mariages se font lorsque le froid force les hommes à quitter les champs et à se retirer dans les maisons, dont ils bouchent les fentes et où ils rentrent le feu. Et les accordailles se font à la saison des semailles et ce sont des unions dans les herbes au moment où les plantes poussent et pour les faire pousser, sans doute, comme on croit encore au Kouang-si. A cette saison favorable, la vertu magique de quelque parfum suffisait à écarter tout danger des unions sexuelles, sanctifiées par la fête même dont elles étaient l'acte essentiel, et, après l'interdit estival, il ne fallait plus que quelques rites pour rapprocher définitivement les époux.

- Mais chez les nobles, gens à mana, gens à ancêtres, l'union de deux enfants de familles dont la personnalité est puissante est d'une a utre gravité que celle des gens de rien. Pour la consacrer, il faut des rites solennels qui atténuent l'opposition des sexes et dont la force mystique mette l'épousée en état de collaborer au culte familial. Tandis qu'au milieu des champs les fiancées paysannes coopéraient au renouveau, auprès des autels domestiques les fiancées nobles s'initiaient au culte qui rend les ancêtres immortels. La licence sexuelle préparait les unes, une sévère claustration les autres, au mariage redoutable.

Mais la vertu de l'empereur se propagea et pénétra les moars du peuple. En même temps que les rites impériaux assuraient à eux seuls In prospérité de la nature, le vulgaire apprit des Seigneurs les règles de la vie domestique. Des fonctionnaires en tournée veillèrent à ce que les sexes vécussent séparés ; selon l'usage noble, et à ce que maris et femmes ne fussent réunis quà la mort ; car lia mort supprime les différences sexuelles et met l'épouse au rang des ancêtres. L'union paysanne, dans les champs, ne fut plus considérée comme un rite de mariage, et le nom dont on la nommait servit à désigner l'union avec les concubines du dernier rang. Les fêtes du printemps passèrent pour d'immorales et grossières pratiques ; tout au plus, en resta-t-il, au deuxième mois, le mariage, en foule et par ordre, des célibataires trop vieux.

Et maintenant, les commentateurs. expliquent que, sill se trouve des chansons printanières dans le Che king, c'est que Confucius, pour inspirer aux princes le dégoût du vice, voulut y admettre des chants obscènes. Encore les y a-t-il admis et nous pouvons, dans son anthologie qui passe pour savante (95), saisir la poésie populaire à sa source même, quand elle naît des fêtes sacrées. 
Marcel GRANET - Coutumes matrimoniales de la Chine antique 22

$*$

** 


\section{- APPENDICE}

\section{Note sur les rapports entre les usages nobles et les usages populaires}

Il y aurait lieu d'insister sur la double lune de miel dans les familles nobles. Je ne puis le faire ici : il s'agit d'un usage seigneu rial et il ne peut être exposé qu'après l'analyse du chapitre du mariage du $\mathrm{Yi} l i$ et de nombreux passages du $\mathrm{Li} \mathrm{ki}$. Je réserve donc cette étude. Ce que je veux montrer dans cette note, c'est comment cette coutume seigneuriale fait sentir la signification de l'interdit estival et voir le pas sage entre les usages nobles et les usages populaires, qui, les uns et les autres, sinspirent des mêmes sentiments de fond.

Pendant trois mois, la jeune fille noble se prépare à son rôle de maitresse de maison et elle vit en recluse (voir plus haut) ; - l'apprentissage fini, elle offre un sacrifice végétal à ses ancêtres : alors elle sort de sa famille, et c'est la pompe nuptiale. Puis, pendant trois mois, elle subit une sorte dinterdit ; ce stage fini, elle offre un sacrifice végétal aux ancêtres de son mari et entre ainsi dans leur famille ( $\underline{\text { Li ki}}$, Couv., I, $\bullet{ }_{429}$ ). Avant la pompe nuptiale on l'appelle : la fiancée, ou la fille; après, on l'appelle : la femme ou mieux : la femme qui est venue ; enfin, après l'of frande, on l'appelle femme, au sens plein du mot. Elle est alors intégrée à sa nouvelle famille; elle sera enterrée dans son cimetière ; les parents de son mari prendront le deuil pour elle.

Mais ce stage de trois mois n'est-il pas autre chose qu'un procédé d'intégration succédant à un procédé de désintégration ? En fait, la parenté entre le mari et la femme, bien que le repas communiel des noces les ait apparentés, n'est parfaite qu'après le troisième mois, puisqu'alors seulement le mari, lui aussi, porte le deuil de la femme avec la rigueur qui convient.

- Toute une école de commentateurs insistent sur le fait que le mariage n'est parfait qu'au bout du troisième mois. [On trouvera tous les éléments de la discussion au $1.423^{\mathrm{e}}$ chap. du HTKKSP. La question y est traitée fort confusément et, selon l'usage, incidemment, à propos d'un problème qui intéresse la critique chinoise: les [] et autres seigneurs ont-ils des rites particuliers de mariage ?] On attire notre attention sur quatre pièces du Che king ; la première du Tcheou nan, et trois pièces du Chao nan qui se suivent, la $2^{\mathrm{e}}$, la $3^{\mathrm{e}}$, la $4^{\mathrm{e}}$. Elles forment, nous dit-on, un groupe, et ont rapport à l'offrande que fait aux ancêtres la jeune épouse en se présentant à eux ; dans la première chanson il s'agit de la femme d'un empereur, dans la deuxième de celle d'un prince féodal, dans la troisième et la quatrième de celle d 'un grand officier. Lecture faite, cette opinion ne paraît pas douteuse. Elle est cependant 
contestée pour la première pièce du Tcheou nan [Cf. Fêtes et Chansons...,

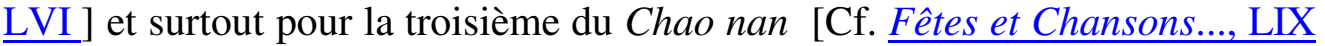
]; la grande raison donnée pour cette dernière est que la préface ne dit pas qu'il s'agit de sacrifice ; mais la raison principale est que, si l'opi nion est admise, elle rend difficile l'interprétation de cette chanson.

La chanson se compose de trois strophes de sept vers dont les cinq derniers forment refrain. Les deux premiers vers des deux dernières strophes parlent de la cueillette des plantes à offrande ; les deux premiers vers de la pièce disent ; «La sauterelle des prés crie, la sauterelle des coteaux saute. » Pour Tcheng K'ang -tch'eng, ceci veut dire : "la sauterelle des coteaux répond à l'appel de celle des prés »; et il y voit une allusion aux temps de fêtes et d'amour du printemps. L'idée des sauterelles évoque en effet celle de l'union sexuelle (voir les commentaires de la $5^{\mathrm{e}}$ pièce du Tcheou nan [Cf. Fêtes et Chansons..., $\underline{\mathrm{VI}}])$. Mais, après un tel exorde, que veut dire le refrain :

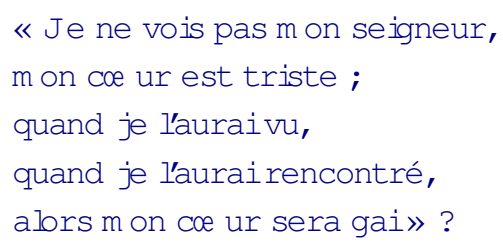

Tcheng ne croit pas que le début des derniers couplets fasse allusion aux sacrifices du troisième mois et, pour lui, une interprétation s’impose : La jeune femme vient de loin à la maison nuptiale; elle cueille des fleurs en chemin, et si son cour est triste, c'est qu'elle a quitté ses parents et a quelque inquiétude sur son sort. Cette explication ne fait pas très bien comprendre les vers du début, ni le refrain ; pourquoi la jeune femme sera-t-elle gaie dès qu'elle aura vu son seigneur?

Pour Tcheng, dans l'expression "voir son seigneur », [] se rapporte à la présentation des fiancés et au repas communiel; quant au mot que j'ai provisoirement traduit par « rencontrer» il se rapporte à la consommation du mariage. Le sens du mot [], fixé par une citation du Yi king, étant celui de rapports sexuels, le refrain devient fort clair: La femme est triste de ne pas voir son mari et ne le sera plus dès qu'elle couchera avec lui ; on comprend que la chanson qui a pareil refrain fasse, à son début, allusion aux amours des sauterelles. Mais alors, les parents quittés passent au moins au second plan; le sentiment essentiel, c'est le désir que le mariage soit consommé.

Le difficile est d'expliquer la cueillette des plantes : Tcheng ne l'explique pas du tout; et il semble bien qu'on ne la puisse comprendre que par le rapprochement avec les deux chansons voisines et la première du Tcheou nan. Mais alors il s'agit du sacrifice aux ancêtres ; la femme est entrée depuis trois mois dans la maison du mari et le mariage n'est pas consommé.

Le gros ennui pour les auteurs qui veulent que le mariage ne soit parfait qu'au troisième mois, c'est que le $Y i$ li, au chapitre du « Mariage des nobles », nous donne de tels détails quill faut bien croire que les époux couchaient 
ensemble dès le premier soir. Et c'est ici qu'on fait intervenir l'idée qu'il y avait des rites spéciaux pour les grands officiers et autres seigneurs. Mais alors, comment comprendre que pour les simples nobles le mariage aussi ne fût parfait qu'après la présentation aux ancêtres ? ( $L i k i$, Couv., I, $\bullet_{429}$, ce texte est très discuté ; je ne puis l'étudier ici.)

- Je ne vois qu'un moyen de rendre compte de tous les faits. La jeune fille noble, accordée par entremetteur, et que rien encore n'a rapprochée de son époux, s'unit à lui après un repas commu niel, qui identifie leurs corps ( $L i k i$, Couv., II, $\bullet{ }_{613}$ ), puis elle en est séparée, et pendant trois mois d’interdit, « ne le voit plus » et n'a plus de rapports sexuels avec lui. Alors la jeune femme est pleine de tristesse, exactement comme la jeune fille de la première chanson du Pin fong, qui, lorsque le printemps était déjà avancé, pensait à la réunion définitive avec son fiancé. La saison de purification écoulée, elle offre le sacrifice végétal, devient épouse au sens plein, et le mariage est parfait .

Si mon hypothèse est juste, après un premier contact consacré par des rites aussi favorables que pouvait l'être la .fête du prin temps, une période de séparation écartait les derniers dangers. Et la double lune de miel féodale correspondrait alors exactement à l'interdit est ival.

Quoi qu'il en soit - et qu'on admette que l'interdit de trois mois après une première union est un fait général, ou que l'on croie que, dans les hautes classes seigneuriales seulement, la consommation du mariage était retardée de trois mois, - il reste qu'après une cérémonie religieuse qui fait des époux «deux moitiés unies », un temps de séparation était encore nécessaire. Cette coutume ne surprendra pas les ethnographes; je rappellerai seulement l'exemple Lolo, cité plus haut, et aussi la coutume védique de la chasteté perdant les trois nuits qui suivent le mariage (Oldenberg, Relig. du Veda, p. $351)$.

Je croirais volontiers que pareil usage était général dans la Chine antique. Mais, comme chez les Lolo, 1a période d'interdit devait être courte et ne pas se prolonger après le temps où la jeune femme est assez pure pour se présenter devant ses beaux parents (c'est-à-dire le troisième jour chez les Lolo, et sans doute en Chine, puisque le troisième jour l'épousée noble se sert de l'escalier réservé à la maîtresse de maison.

- Cette période de trois jours est devenue dans les mariages aristocratiques une période de trois mois : on sait que, dans le comput religieux du temps, c'est le nombre plutôt que l'unité d e mesure qui importe. Or on avait à rapprocher des personnalités plus puissantes, et les fiançailles, faites par entremetteur, n’avaient pas préparé les épousailles ; enfin, pour être présentée aux ancêtres, la femme doit être dans un état de pureté plus parfaite que pour la présentation aux parents vivants, et, avec les progrès du culte familial, seule l'offrande dans le temple pouvait sembler suffisante à parfaire le mariage. 
Même l'idée que le mariage a pour fin de sanctifier l'union sexuelle dut être primée par l'idée qu'il a pour fin de donner une servante aux ancêtres. Ce qui resta de l'interdit, ce fut l'inter diction de sacrifier et de travailler, et non pas les interdictions sexuelles, parce que l'intégration de la femme dans la famille finit par paraître plus importante que son assimilation au mari.

Ainsi s'explique le passage des rites paysans aux rites aristo cratiques. 


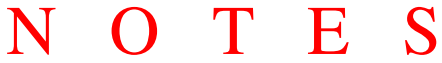

[css : rappel : on circule entre les traductions de M. Granet (Fêtes ...) et du P. Couvreur (Cheu king) en cliquant sur le signe • en titre de la chanson. Un clic sur le nom de la chanson dans Fêtes... envoie sur le site très recommandé de l'afpc contenant les mêmes traductions, ainsi que le texte chinois, et la traduction anglaise de J. Legge]

(1) Je renverrai toujours pour le Che king à la traduction du P. Couvreur. Elle a le très grand mérite de donner l'interprétation traditionnelle. Je cite les commentaires d'après l'édition classique. Je donne pour qu'on y retrouve les pièces, la section à laquelle elles appartiennent et leur numéro d'ordre. J'ai utilisé les travaux modernes contenus dans les collection (1 -2) HTKK et HTKKSP. J'ai pris le texte du petit calendrier des Hia dans les chap. 573-578 du HTKKSP.

(2) Sauf sur quelques points, pour préciser, ou établir des transitions, j'ai laissé de côté tout ce qui dans le Che king se rapporte aux usages aristocratiques. Je crois qu'à l'époque où ces chansons ont été recueillies, une conception particulière du mariage existait déjà dans les classes nobles celle que nous connaissons par les rituels et que les auteurs chinois croient être primitive (voir plus loin).

() Le commentaire est de Wang Sou.

(4) Che king, Pei fong, $\mathrm{n}^{\circ}$ 9, Couv., p. • ${ }_{38}$. [Cf. Fêtes et Chansons..., L, v. 11-12.]

(5) Che-king, Wei fong, ${ }^{\circ}$ 4, Couv., p. • 67 . [Cf. Fêtes et Chansons..., LXVI, v. 9-10.]

(ㅁ) Nous allons revenir sur ces textes.

(7) La traduction que je donne du caractère [] est provisoire. C'est celle qui me paraît correspondre à l'idée que se faisait de ce mot le commentateur cité.

() Il faut rappeler que les saisons chinoises ne commencent pas aux équinoxes et aux solstices, mais que ces dates en marquent le milieu.

(9) Tcheng K’ang-tch'eng mérite sa réputation. Nous verrons qu'il est le commentateur qui a eu le sens le plus vif du problème; il a compris que les rites du mariage se divisent en deux groupes séparés par un long espace de temps.

(10) Couv., p. • 10. [Cf. Fêtes et Chansons..., I, préf.]

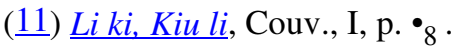

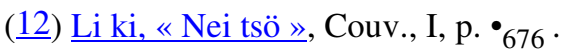

(13) On peut voir de quelle prudence il est besoin pour utiliser les notes des commentateurs : chacun d'eux a une théorie, et l'interprétation qu'il d onne d'un texte particulier est solidaire de celle qu'il a adoptée pour un groupe fort important de textes, souvent très différents ; pour se rendre compte des faits, il faut donc grouper ces interprétations de détail et retrouver la théorie de chaque école de commentateurs. Ce n'est qu'à cette condition qu'on peut utiliser pour l'interprétation des textes et leur traduction les travaux de la critique chinoise.

(14) La $9^{\mathrm{e}}$ pièce du Pei fong, Couv., p. 38 . [Cf. Fêtes et Chansons..., L]. Voir plus loin .

(15) On reconnaît la théorie formulée plus haut. Les deux rites sont les deux premiers des fiançailles. 
(16) Li ki, Kiu li, Couv., I, p.• 31 .

(17) J'ai déjà dit que je fais aussi la distinction entre les usages nobles et populaires. Je n'ai pas à tenir compte des textes du Tsouo tchouan puisqu'il ne s'agit ici que des coutumes populaires.

(18) On en verra un exemple plus loin.

(19) Dans le Kia yu, le duc Ngai de Lou s'étonne que les rites ordonnent d'attendre 20 et 30 ans pour se marier. Confucius lui explique que c'est mal comprendre et que les rites fixent l'âge limite et non l'âge obligatoire du mariage.

(20) Voir par exemple les commentaires de la $9^{\mathrm{e}}$ chanson du Pei fong; on en trouvera plus loin quelques-uns.

(21) Les auteurs chinois croient à un sentiment inné de la morale : les hommes ne pèchent contre les rites que lorsque l'état social est mauvais. En même temps ils croient fermement que la civilisation s'est répandue peu à peu et que c'est l'action gouvernementale qui a tiré les hommes de l'état sauvage.

(22) Pour le travail que je veux faire il est nécessaire que le rythme de la chanson chinoise (qui seul décèle les correspondances) soit conservé dans la traduction. J'ai donc traduit en phrases de 6 ou 8 syllabes françaises les vers de 3, 4, ou 5 caractères. La densité du chinois ne rend pas le travail facile. Je n'ai pas hésité pour me tirer d'affaire à employer la syntaxe raccourcie de nos vieilles chansons. La physionomie que cela donne à la traduction est loin de trahir celle de l'original. Mon principe est donc de traduire vers pour vers. Je n'ai dédoublé le vers chinois que lorsque je pouvais le faire sans cacher aucune correspondance, et quand la clarté et le rythme de la traduction française le rendaient nécessaire ; j'ai soigneusement fait passer dans mon texte les retours d'expressions qui indiquent que deux vers se font pendant.

(23) Bonifacy, Etude sur les coutumes et la langue des Lolo et des La-qua du haut Tonkin, B. E. F. E. O., VIII, p. 531 et suiv. Voir aussi les notes à la traduction des «Barbares soumis du Yunnan », ibid., p. 333 et suiv. [Cf. Fêtes et Chansons..., App. III ].

BEAUVAIS, Notes sur les coutumes des indigènes de la région de Long-tcheou, B. E. F. E. O., VII, p. 265 et suiv. [Cf. Fêtes et Chansons..., App. III ]. Voyez aussi T'ANG TSAI-FOU, Le mariage chez une tribu aborigène du Sud-Est du Yun-nan, T'oung pao, 1905, p. 596-598.

(24) Il se trouve que la première traduction que je donne est celle où je parais le plus infidèle à mes principes. Voici pourquoi : La chanson se compose de deux couplets de quatre vers et d'un refrain de huit vers. Le refrain est très dense, très riche en faits; de plus ces huit vers ne sont pas distribués en deux groupes de quatre vers mais en trois groupes; le premier (deux vers) donne le début du chant dialogué, le deuxième (trois vers) nous montre les jeunes filles qui insistent, le troisième (trois vers) nous conte délicatement la partie essentielle de la fête. J'ai donc rendu chacun de ces trois groupes, par un groupe de quatre phrases. On constatera que les parallélismes d'expression sont quand même conservés (« là-bas si nous allions »). J'ai supprimé le mot cueillir au quatrième vers pour insister sur le caractère [] qui établit la correspondance avec le premier vers. J'ai préci sé d'après les commentaires le sens des deux [].

(25) Seul un commentateur moderne fait remarquer que, puisque Tcheng lui-même rapproche les réunions champêtres que décrit la chanson du texte connu du Tcheou li, c'est qu'elles sont parfaitement normales et régulières, HTKK, drap. 335, p. $18 \mathrm{r}^{\circ}$ (20).

(드) Dans une autre pièce amoureuse ( Kouo fong, section Tcheng, ${ }^{\circ}$ 2, Couv., p. $\bullet_{145}$ ), les jeunes gens se donnent aussi une plante odorante dont il est dit (voir HTKKSP, chap. 428, à cette pièce) qu'on s'en sert pour faire descendre les divinités.

(27) Ces sachets se nomment [] pour les femmes (Couv., Li ki, I, 622) et [] pour les jeunes filles (Couv., I, 624). La concubine (Couv., Li ki, I, 661) se purifie, se rince la bouche, lave 
ses vêtements, etc., et attache le sachet). Cf. la deuxième strophe de la $8^{\mathrm{e}}$ chanson du $\underline{W e i}$ fong, Couv., p. $\bullet^{73}$, « est-ce que je manque de parfum et d'eau ? mais pour quel maître me parer?»

(28) Voir H. T. K. K. S. P., chap. 423 aux notes sur la pièce en question.

(29) Une jolie chanson qui s'appelle la vierge sage (la $17^{\mathrm{e}}$ du Pei fong, Couv., p. ${ }_{49}$ ) [Cf. Fêtes et Chansons..., XXXIX ], nous fait sentir l'importance de ce gage d'amour : "Plante qui viens des pâturages - étrange et belle en vérité - ta beauté, ce n'est point la tienne : tu es le don d'une beauté ». Cette pièce me suggère que le don du gage pourrait bien être mutuel, contrairement à l'opinion de Tcheng K'a ng-tch'eng que j'ai suivi pour traduire la chanson précédente.

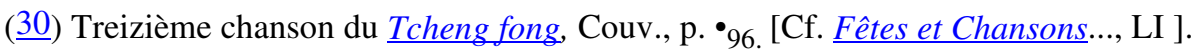

(31) Pour se convaincre des méfaits de l'interprétation morale, on n’a qu'à comparer la traduction du P. Couvreur [cf. note 14], qui a fidèlement suivi la tradition, à la mienne qui est littérale. Le développement est pourtant clair. «Voici que vient la crue des rivières et je passerai l'eau, avec l'ami de mon choix, en soule vant ma jupe, ou en la troussant si l'eau vient plus haute, ou bien en voiture, ou même en bateau. » Les vers 7 et 8 sont les plus maltraités par l'interpréta tion traditionnelle.

(32) La correspondance deux à deux des vers du deuxième couplet indique nettement que le chant de l'oiseau et la crue des rivières sont des événements liés, contemporains. Le premier et le deuxième vers commencent tous deux par « voici que... et que ».

(33) Les commentateurs n'ont pas aperçu l'allusion — le mot pourtant est le même (voir le commentaire de Tcheng au Yi li). Aussi ce qu'ils ont voulu expliquer, ce n'est pas la courge, mais les feuilles amères. Ils croient que l'on veut dire : la courge est inutilisable ; en effet, ajoutent-ils, si elle a des feuilles amères, on ne peut la manger ou s'en servir pour la natation. S'il faut absolument expliquer ces feuilles amères, je dirai : la calebasse ayant encore ses feuilles amères, on ne peut l'utiliser aux noces ; le temps des noces n'est pas venu. Mais je préfère voir dans l'expression, comme dans celle «oies sauvages qui se répondent» une espèce d'épithète homérique.

(34) Voir Yi li. L'expression « qui se répondent» fait voir une des raisons qui, pour les Chinois, expliquent que les oies sauvages aient été choisies comme présent de noces: ces oiseaux volent, disent-ils, par couples, la femelle un peu en arrière, comme il convient, et quand le mâle crie, elle répond. Le P. Couvreur traduit fort bien en latin consonance, mais il fait un contre-sens en français : (à la voix harmonieuse), cf. Couv. p. 5.

(35) Voir la traduction de la chanson qui suit, et aussi, par exemple, la chanson I du Chao nan, Couv., p. • 16. [Cf. Fêtes et Chansons..., IX ].

(36) On a vu que c'est de ce vers que s'autorise le Kia yu. Il importe quelque peu à l'hypothèse que nous formulerons quil ne s'agisse pas de la pom pe nuptiale; car nous la plaçons à l'automne. L'opinion de Tcheng K'ang -tch'eng nous est donc précieuse : il ne le gênait en rien de voir là une allusion à la pompe nuptiale, car pour lui les mariages se font précisément à l'époque que désigne le vers sui vant « avant que les glaces ne soient fondues ». Il est donc significatif que de l'emploi syntaxique du mot [] il ait déduit qu'il ne s'agissait pas de la pompe nuptiale, mais d'un rite antérieur, préparant le mariage.

(37) Remarquons que le caractère symétrique [] s'emploie d'une poule. Voir Couvreur, Dictionnaire.

(38) Il faut entendre dans leur force les caractères [] qui se balancent ; pour traduire en toute rigueur, il faudrait dire : la perdrix cherche le mâle qui fera la paire avec elle — j'attends l'ami qui fera la paire avec moi. On trouve (au $\mathrm{n}^{\circ} 5$ de la $1^{\mathrm{e}}$ section du Siao ya) le vers: 
(l'oiseau) cherche son compagnon en chantant. Un mot est employé dans notre chanson à propos de la jeune fille, d'autres mots à propos de la perdrix.

(39) $\mathrm{N}^{\circ} 3$ du Pin fong, Couv., p. ${ }^{167}$. [Cf. Fêtes et Chansons..., VIII ].

$(\underline{40}) \mathrm{N}^{\circ} 1$ du Pin fong, Couv., p. • 160 . Cf. Siao ya, «Lou ming», n 6. [Cf. Fêtes et Chansons..., XXI ].

(41) Couv., Li ki, I, p. $\bullet_{310}$. Notons en passant, à propos du pêcher, que la floraison des arbres est aussi un thème des chansons d'amour. Voir le $\mathrm{n}^{\circ} 6$ du Tcheou nan, Couv., p. ${ }_{10}$ • [Cf. Fêtes et Chansons..., I].

(ㄴ) Couv, p.•13. [Cf. Fêtes et Chansons..., XLVI ].

(433) B. E. F. E.- O., VIII, p. 338.

(44) Couvreur, p. ${ }^{120}$. Voir plus loin p. 35, n. 3. [Cf. Fêtes et Chansons..., LXI ].

(느) Voir ce qu'en disent les commentateurs modernes H.T.K.K.S.P., chap. 426, à la chanson que je viens de citer.

(46) Voir Ts ï fong, $\mathrm{n}^{\circ}$ 6, Couv., p. ${ }_{107}$; Pin fong, $\mathrm{n}^{\circ}$ 4, Couv., p. $\bullet_{170}$ et Siao ya, sang hou, $\mathrm{n}^{\circ} 3$, Couv., p. ${ }^{2} 293$.

(47) Chao nan, $\mathrm{n}^{\circ} 2$, Couv., p. ${ }^{17}$ •

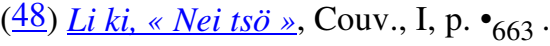

(49) Je trouve une espèce de confirmation dans les deux emplois du mot qui désigne ces fagots d'herbe ou de bois. Pour dire qu'un je une homme du peuple est déjà âgé (le contexte fait voir que cela veut dire majeur et par conséquent en âge de se marier), on dit qu'il peut porter un fagot ( $\mathrm{Li} \mathrm{ki}$, Kiu li, Couv., I, p•• $\left.{ }_{98}\right)$. D'autre part, quand un offici er s'excuse de ne pas participer à la cérémonie religieuse du tir à l'arc, il dit : "Un tel à l'impureté du fagot porté » (Li ki, Kiu li, Couv., I, p.• 73 ). Ce texte est complété par un texte du Kiao t'ö cheng ( $\underline{\text { i }}$ ki, Couv., I, $\left.{ }_{585}\right)$ : Un officier invité à tirer de l'arc s'excuse en alléguant une impureté « selon le sens qu'a l'arc suspendu » (à la naissance d'un enfant).

$(\underline{50})$ Je traduis par biche un caractère qu'on définit, « espèce de cerf timide ». Je garde le mot biche au $6^{\mathrm{e}}$ vers; tous les commentateurs sont d'accord : ces animaux désignent tout simplement le cerf dont la peau est un présent rituel.

(51) Le [] du [] qui décrit la présentation des peaux de cerf ne parle pas de l'herbe dont on les enveloppait.

(52) Reconnaissons en passant le thème des fagots.

(53) Les commentateurs attribuent même sens à [] et [].

(54) J'ai rendu jade et serviette par des équivalents, afin de faire sentir, à peu près, la signification qu'ils ont en chinois, et que leur traduction littérale ne fait point paraître.

(55) Je dédouble ce vers afin de rendre le [] qui marque un si joli mouvement dans le vers chinois.

(므) $\underline{\text { Li ki }, ~ « N e i ~ t s o ̈ ~ », ~ C o u v ., ~ I, ~}{ }_{663}$.

(ㄱ) Pin fong, ${ }^{\circ} 4$, Couv., $\bullet 169$.

(모) Yi li, La mère dispose la ceinture et attache la serviette. 
(모) Yi li.

(60) C'est à cause des derniers vers que cette fille « qui a la pureté du jade » est qualifiée de «fille chaste» par les Commentateurs. Je le veux bien : mais dans le même sens que la jeune fille de la chanson citée plus haut est appelée « vierge sage ». Qu'elles vivent dans la retraite et qu'elles observent les rites qui leur défendent de s'unir, avant l'automne, à leurs amis, cela n'empêche pas qu'elles ne se rappellent les réunions de printemps ni qu'elles n'y aient échangé des fleurs.

(61) Une autre chanson de la mal mariée rappelle de manière analogue le passage de la rivière ; et, ce qui est significatif, on y voit reparaître les expressions mêmes des chansons de la courge et de la rivière Han (Pei fong, 10, Couv., p. 39, [Cf. Fêtes et Chansons..., XLIX ]).

(62) On se rappelle que les La-qua de même village ne chantent pas ensemble ; c est une conséquence de la règle d'exogamie.

(63) Comme chez les Lolo, l'entremetteur n'est pas un manieur qui assortit les couples. Il est l'ambassadeur d'une famille à l'autre. Mais, avant, les jeunes gens s'entendent. Une chanson du $\underline{T s}$ i fong (Couv., 107) marque bien le procédé : les jeunes gens avertissent les parents de leurs intentions, puis l'entremetteur est envoyé.

(64) Voir Tcheng fong, $\mathrm{n}^{\circ} 20$, Couv., p. • ${ }_{101}$ [Cf. Fêtes et Chansons..., X ] .

(65) Voir Chao nan, ${ }^{\circ}$ 6, Couv., p. ${ }_{20}$ [Cf. Fêtes et Chansons..., XI ].. Sur le dépôt de la rosée et de la gelée blanche, voir aussi la pièce $\mathrm{n}^{\circ} 4$ du Ts'in fong, Couv., ${ }_{137}$ [Cf. Fêtes et Chansons..., LIV ].

(66) Aucun texte, et pour cause, ne nous dit que ces réunions printanières étaient destinées à assurer la fécondité du sol. Mais il n'est pas douteux qu'il y ait eu pour les anciens Chinois un lien entre les relations sexuelles et la germination des plantes. Ainsi l'impératrice garde les semences (destinées au champ impérial) dans le gynécée comme présage d'une belle descendance, et au début du printemps à la tête des femmes des 6 palais, elle fait germer les différentes graines : voir Tcheou li, chap. 7, et le commentaire de Tcheng.

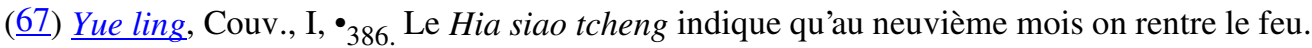

(68) Pin fong, ${ }^{\circ}$ 1. Couv., • ${ }_{160}$ [Cf. Fêtes et Chansons..., XXI ]., particulièrement les strophes 5,7 et 8 . Voir aussi T'ang fong, $\mathrm{n}^{\circ} 1$, Couv., $120:$ le grillon domestique est lié à l'idée du repos des laboureurs et aux réjouissances qui marquent la fin des travaux ; le chant du grillon dans les maisons est du neuvième mois, la fête du repos des laboureurs du dixième ; il est dit ici : «l'année touche à sa fin »: ces difficultés de calendrier embarrassent les commentateurs : on verra comment nous en rendons compte plus loin.

(으) B. E. F. E. O., VII, p. 215-216.

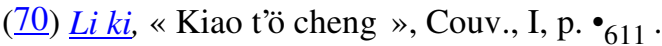

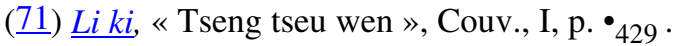

(72) $\underline{L i k i}$, «Kiao t’ö cheng », Couv., I, p. ${ }_{611}$.

(73) Pin fong, $\mathrm{n}^{\circ} 1$, Couv., p. $\bullet_{160}$ [Cf. Fêtes et Chansons..., XXI ].. Le sentiment d'anxiété des époux s'exprime admirablement dans la pièce intitulée « le fagot lié », T'ang fong, ${ }^{\circ} 5$, Couv., p. • ${ }_{124}$ [Cf. Fêtes et Chansons..., LXI ].: «En fagot sont liées les branches ; - les trois étoiles sont au ciel. — Quelle soirée que ce soir ci ! Maintenant voici mon homme, hélas de moi ! hélas de moi ! — Comment sera-ce avec cet homme ?» 
(74) - Notons, ce que nous dit le Yue ling, qu'après la fête des mariages, un héraut passait avec sa clochette à battant de bois : il interdisait les rapports sexuels, qui à ce moment auraient mis l'État en danger.

(드) $\underline{\text { Li ki, }}$ « Li yun », Couv., I, p • 510 .

(드) Che king, Couv., p. $\bullet_{42}$ et $\bullet_{113}$.

(77) Le commentaire du $\mathrm{n}^{\circ} 1$ du Wei fong. Alors seulement l'épou sée mérite pleinement le nom de «femme ». Voir la Note placée en appendice.

(78) On trouvera sur ce point un supplément d'information dans la Note placée en append ice.

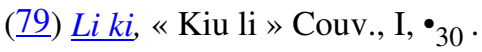

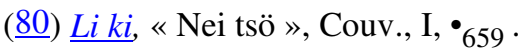

(1) Wang fong, Couv., p. 83 [Cf. Fêtes et Chansons..., XLIII ].

(르) $\underline{\mathrm{Li} k i}$, « Kiu li », Couv., I, ${ }_{30}$ •

(3ㅡ) Par exemple lorsque les critiques chinois rencontrent des expressions telles que «quand l(homme s'en va chercher femme ", ils essaient aussitôt de voir auquel des rites de leur temps on peut la rapporter et, selon quỉls y voient un rite de fiançailles, ou la cérémonie principale, ils édifient les théories que nous connaissons sur l'époque des mariages. En fait, dans les chansons du Che king, l'emploi du mot [] ou même de l'expression « cette fille se marie », ne suffit pas à prouver que le chant soit un chant d'hyménée ; rites printaniers et pompe nuptiale sont des parties importantes des rites matrimoniaux et, quand on procède à l'un de ces rites, la chanson chantée peut fort bien rappeler l'autre.

(4) La comparaison du Tcheou li et du Hia siao tcheng révèle le caractère administratif du premier. Le calendrier des Hia dit simplement « on réjouissait en grand nombre les jeunes gens et les jeunes filles ». Ce qui même peut se traduire (vu l'usage des inversions dans ce texte) « au deuxième mois de printemps, garçons et filles en grand nombre se réjouissaient ». $\mathrm{Au}$ contraire, le Tcheou $\mathrm{Li}$ affirme l'intervention administrative ou bien par parti pris de rédaction, - ou bien, plutôt, parce qu'en effet on avait régularisé administrativement une coutume impossible à détruire.

(5) Il y en avait d'analo gues à l'époque des Han. Voir Annales principales : quand une fille n'est pas mariée après 15 ans et avant 30 ans (ses parents doivent payer une amende de 5 souan. D'après la loi des Ts'ing une esclave -servante doit être mariée par son maître avant 23 ans. V. P. Hoang, Mariage chinois, p. 226. Il y aurait beaucoup à dire sur l'idée qu'il importe à l'Etat que les filles soient mariées. Mais ce n'en est pas le moment.

(6) C'est parce quill dépend du calendrier que le mariage ressort it à la juridiction d'État. Voir Chao nan $\mathrm{n}^{\circ} 6$, Couv., p. ${ }_{20}$ [Cf. Fêtes et Chansons..., XI ].

(7) Voir Pin fong, ${ }^{\circ}$ 1. Couv., p. • 160 [Cf. Fêtes et Chansons..., XXI ].

(요) Déjà le neuvième mois est postérieur au solstice d'automne.

(9) On voit combien Wang Sou a de difficulté à soutenir sa théorie du mariage en automne-hiver (saisons yin) puisqu'il est obligé de faire commencer la saison des Mariages après le solstice d'automne (près de deux mois après le début officiel de l'automne) et de la faire finir au deuxième mois de printemps, deux mois après qu'on est officiellement entré dans la saison yang.

(90) Couv. I, 396. Voir la description au $\underline{n}^{\circ} 1$ du Pin fong, strophes 7 et 8.

(91) Aussi les théories de Wang Sou sont-elles plus distantes des faits que celles de Tcheng K'ang -tch'eng. Mais comme, ni pour l'un ni pour l'autre, le yin et le yang n'étaient de purs 
principes d'explication astronomique, il reste dans leurs spéculations assez de concret pour qu'on puisse en profiter. De Wang Sou et du Kia yu nous avons tiré l'idée que la saison du travail champêtre s'opposait à celle du repos urbain ; de Tcheng, celle de l'importance des périodes critiques.

(92) Wang fong, $6^{\text {e }}$ pièce, Couv., p. 83 [Cf. Fêtes et Chansons..., XLIII,]. : «j'ai peur du seigneur ; je n'irai plus aux champs m'unir à toi ».

(93) Cette phrase ferait volontiers croire à une coutume régularisée ; les réunions dans les champs n'étaient plus permises qu’à une époque administrative ment déterminée.

(94) Couv., I, p. ${ }^{6} 676$ •

(95) On connaît la prédilection des écrivains chinois pour les allusions littéraires qui consistent à encadrer une sentence ancienne dans un développement nouveau, et pour les phrases accouplées, qui sont des sentences strictement balancées et où les mots symétriques se correspondent rigoureusement. Et ce sont des procédés savants. Ils sont, aussi, essentiels à la poésie populaire du Che king. Les chansons s'empruntent, dans les termes mêmes, des thèmes les unes aux autres, et les couples de vers ne sont pas rares où les caractères, deux à deux, se font pendant. 
Notes - - 
Nom du document : coutumes_matrimoniales.doc

Dossier :

C:ICSSIEnvoi021204lgranet_marcel

Modèle :

C:IWINDOWS $\backslash$ Application

DatalMicrosoft $\backslash$ Modèles\Normal.dot

Titre :

Sujet :

Auteur :

Coutumes matrimoniales de la Chine antique

Mots clés :

série Chine

Marcel Granet

chinoise, Chine ancienne, Chine antique, Chine classique, ethnologie de la Chine, mythologie chinoise, sociologie de la Chine, confucius, civilisation chinois, religion chinoise, ancient China, sinologie, a

Commentaires : iences_sociales/index.html

http://www.uqac.uquebec.ca/zone30/Classiques_des_sc

Date de création : $\quad$ 18/11/04 13:29

$\mathrm{N}^{\circ}$ de révision : $\quad 5$

Dernier enregistr. le : 01/12/04 21:52

Dernier enregistrement par : Pierre Palpant

Temps total d' édition4 Minutes

Dernière impression sur : $\quad$ 05/12/04 12:03

Tel qu' à la dernière impression

Nombre de pages :

34

Nombre de mots : 13458 (approx.)

Nombre de caractères : 76715 (approx.) 